\title{
Absence of metallicity in Cs-GaAs(110): A Hubbard-model study
}

\author{
Z. Gedik and S. Ciraci \\ Department of Physics, Bilkent University, Bilkent 06533, Ankara, Turkey \\ Inder P. Batra \\ IBM Research Division, Almaden Research Center, 650 Harry Road, San Jose, California 95120-6099
}

(Received 3 February 1992)

\begin{abstract}
Using an approximate solution of the Hubbard-model Hamiltonian, we are able to establish that the Cs-GaAs(110) system becomes a Mott insulator at submonolayer Cs coverages. We also provide a consistent interpretation of electron-energy-loss and scanning-tunneling-spectroscopies data. The correlation effects are important for this system with an estimated correlation energy of $0.4 \mathrm{eV}$.
\end{abstract}

The adsorption of alkali-metal (AM) overlayers on semiconductor surfaces has attracted considerable interest recently. ${ }^{1-7}$ The filling of the active dangling-bond surface states by the weakly bound valence electron of the adsorbed AM atoms underlie several properties, such as bonding, surface reconstruction, metallization, and collective excitations. The current issues subject to active investigation are the character of the bonding and surface metallization, and the adsorption site at various stages of AM coverage. Numerous observations are consistent with the fact that the properties exhibit significant variations depending on the range of coverage. Total-energy calculations ${ }^{3,7}$ show that potassium can be adsorbed onto at least four different sites on the $\mathrm{Si}(001)-(2 \times 1)$ surface, which are of comparable binding energy, implying that the bonds formed with AM atoms are nondirectional.

Electronic structure and charge-density analysis of several AM-covered semiconductor surfaces indicated that the weakly bound valence-electron of the adsorbed atom is donated to the empty dangling-bond surface state. $^{2}$ In some cases, the dispersion and the charge distribution of this band do not change significantly upon filling with alkali adsorption. Having no unambiguous way to measure the charge transfer, this has been taken as evidence of a strongly ionic bonding of the AM atom with semiconductors. Previous assertions that the bond should be covalent are now reconciled with a partly ionic picture, even for an estimated charge transfer of 0.6 electrons.

Earlier, in the analysis of the stability of reconstruction upon the AM adsorption, we pointed out the strong electron-correlation effect in the filling of the flat surface-state band. ${ }^{8}$ These arguments appear to be valid for the electronic structure of the GaAs(110) surface at submonolayer Cs coverage: It is established that in the clean GaAs(110) surface the surface atoms relax; while the $\mathrm{Ga}$ atoms are lowered relative to their ideal positions, the As atoms are raised. The buckling is, however, reduced upon absorption of an alkali atom. Previous electron-structure calculations based on the local-density approximation (LDA) yielded a finite density of states (DOS) at the Fermi level in the band gap for the half monolayer $(\theta=0.5)$ as well as for the monolayer $(\theta=1)$ coverage of AM. ${ }^{9-11}$ The difference charge-density (i.e., charge density with AM minus that without AM) analysis revealed that the charge due to the adsorbed AM has a dangling-bond orbital character and is strongly localized at the surface Ga atoms. ${ }^{11}$ However, the data obtained from scanning tunneling microscopy (STM), ${ }^{12}$ scanning tunneling spectroscopy (STS), ${ }^{13}$ photoemission electron spectroscopy, ${ }^{14,15}$ and electron-energy-loss spectroscopy (EELS) (Ref. 16) provided evidence that the Cscovered $\mathrm{GaAs}(110)$ surface is insulating for $\theta<1$. Apparently, these experimental results are at variance with the standard energy band-structure calculations predicting metallic DOS.

Earlier, the chain structure revealed by STM at low coverage was identified as the one dimensional (1D), zigzag chain of adsorbed Cs atoms. ${ }^{12}$ Such a chain involves a local $c(2 \times 2)$ surface structure with $\theta=0.5$ coverage. An extended area of adjacent zigzag chains leads to a hexagonal-like structure of Cs atoms with the Bravais lattice vectors of $\left|\mathbf{R}_{1}\right| \simeq 6.9 \AA$ and $\left|\mathbf{R}_{2}\right| \simeq 7.9 \AA$. The coverage $\theta=1$ is supposed to lead to a rectangular structure having the unit cell of the GaAs(110) surface. ${ }^{16} \mathrm{We}$ name this structure as the compressed phase or rectangular structure. Recent current-voltage measurements (STS) (Ref. 13) over various Cs structures on GaAs(110) clearly show that the band gap of the clean surface is 1.4 $\mathrm{eV}$. However, first it decreases to $1.1 \mathrm{eV}$ for $1 \mathrm{D}$ structure at $\theta \simeq 0.1$, later to $0.6 \mathrm{eV}$ for $2 \mathrm{D}$ structure at $\theta \simeq 0.6$. Finally, at the onset of 3D overlayer for $\theta \geq 1$, the band gap diminishes, leading to a conducting state. In compliance with the earlier observations, the $1 \mathrm{D}$ structure is made from zigzag chains, which become wider upon the formation of adjacent chains at increased $\theta$, whereas, recent STM images of 2D phase are identified as five-atom polygons with a local $c(4 \times 4)$ structure. ${ }^{13}$ The local electronic structure of Cs overlayer revealed by STM and STS is in agreement with the interpretation of EELS. ${ }^{16}$ For $\theta \rightarrow 0.5$ the band gap narrows in the EELS spectrum. Two loss peaks (at 0.42 and $1.04 \mathrm{eV}$ ) originating from localized excitations appear only when $\theta>0.5$. Near the saturation coverage at room temperature $(\theta \simeq 0.9)$ a new loss peak appears at $1.14 \mathrm{eV}$. Additional Cs coverage, which takes place only at low temperature, shifts this 
peak to the plasmon energy of bulk Cs. Concomitantly, the band gap is filled by a continuum of excitations.

The evolution of the electronic structure with coverage $0 \leq \theta \leq 1$ is identified by the transition from 1D-2D (Mott) (Ref. 17) insulator to 3D metal. It is argued that the electron density of the overlayer is low, and the electronic structure can be described by many electron models invoking on-site Coulomb energy $U .{ }^{16}$ Because of $U$, the density of states in local (1D or 2D) AM structures on the GaAs(110) surface splits to prevent double occupancy at a given site.

There are various phenomena that give rise to the metal-insulator transition. ${ }^{17}$ For example, a change in crystal structure may create a gap in energy spectrum. In Cs-covered GaAs(110), a local change in the periodic structure is not compatible with such a transition. More complicated transitions are also possible, such as those associated with Neel ordering or exciton formation. The latter is not possible for a metal-covered semiconductor surface since it requires long-lived excitons. It appears that the electron-electron correlation is the likely source of observed transition. It should be noted that the nearest-neighbor distance of the $\theta=0.5$ structure $(6.9 \AA)$ is significantly larger than that of bulk Cs, which is known to be close to the Mott metal-insulator transition. ${ }^{18}$ In this study we investigate the effect of electronelectron correlation in the Cs-adsorbed GaAs(110) surface by using the 2D Hubbard model. ${ }^{19}$ The electronic structure derived therefrom is used to interpret STS and EELS data.

When Coulomb interaction of electrons is introduced, we have to add to the standard Hamiltonian, terms involving four states. Those are two incoming states that interact via electrostatic force and two outgoing states into which the initial electrons are scattered. By expressing the Hamiltonian in terms of localized orbitals (such as Wannier orbitals $\phi_{i}$ ), one can see that the interaction terms having the same four states have the largest magnitude. Thus, all the interaction terms involving different sites can be neglected. This leads to the Hubbard model with the Hamiltonian ${ }^{19}$

$$
H=\sum_{i, j, \sigma} t_{i j} c_{i \sigma}^{\dagger} c_{j \sigma}+\frac{U}{2} \sum_{i, \sigma} n_{i \sigma} n_{i-\sigma},
$$

where $c_{i, \sigma}^{\dagger}$ is the creation operator for an electron of spin $\sigma$ at site $i, n_{i, \sigma}=c_{i, \sigma}^{\dagger} c_{i, \sigma}$ is the number operator, $t_{i j}$ is the transfer integral $\left\langle\phi_{i}|H| \phi_{j}\right\rangle$ from site $j$ to site $i$, and $U$ is the Coulomb repulsion energy between two electrons on the same site, which is given by

$$
U=e^{2} \int d^{3} r d^{3} r^{\prime} \frac{\left|\phi_{i}(\mathbf{r})\right|^{2}\left|\phi_{i}\left(\mathbf{r}^{\prime}\right)\right|^{2}}{\left|\mathbf{r}-\mathbf{r}^{\prime}\right|} .
$$

In the presence of local vibrational excitations, there may arise a negative- $U$ term that renormalizes the Coulomb repulsion. However, in our system, owing to the insulating nature of the metal layer, we assume that electronphonon interactions are not important. Although its exact solution is not known, there has been great progress in understanding the nature of the Hubbard model for the 2D square lattice, due to the widely accepted view that it describes the physics of high-temperature oxide superconductors. For high- $T_{c}$ materials, it has been proposed that $t / U$ ( $t$ being the largest $t_{i j}$, i.e., nearestneighbor hopping, and all the other $t_{i j}$ 's are neglected) ratio may be rather small and therefore the hopping term can be treated perturbatively. In this case, using degenerate perturbation theory, ${ }^{21}$ it is possible to show that the model reduces to the so-called $t-J$ model, which contains terms of the form $-J \mathbf{S}_{i} \cdot \mathbf{S}_{j}$. Here, $\mathbf{S}_{i}$ is the spin operator for site $i$, and $J$ is the exchange integral between sites $i$ and $j$, and it is given by $J=-4 t^{2} / U$. In the $\mathrm{Cs} / \mathrm{GaAs}(110)$ system, the $t / U$ ratio is small; it may be even smaller than that for high- $T_{c}$ compounds. Therefore, all the discussions related to the 2D $t-J$ model may be relevant for the $\mathrm{Cs} / \mathrm{GaAs}(110)$ system also. For our purposes it is enough to know how the correlation effects change the DOS of the system. Here, by a site we mean the localized superorbital formed from the orbitals of Cs and those of the surface atom to which Cs is bound.

An appropriate solution of the energy spectrum of Eq. (1), which reduces to localized orbitals or Bloch states at the proper limits, is provided by the Green-function technique. ${ }^{19}$ For the sake of simplicity, we confine ourselves to the solution at $0 \mathbf{K}$ and we assume that the system is nonmagnetic, thus $\left\langle n_{i \sigma}\right\rangle=n_{\sigma}=\frac{1}{2}$. The last equality follows from the fact that each Cs atom donates one electron to the system. Under those conditions, the density of states $\rho(E)$ in the presence of the Coulomb repulsion is given by

$$
\rho(E)=\frac{1}{N} \sum_{\mathrm{q}} \delta\left[E-I-\frac{I^{2}}{E-I}-\epsilon(\mathbf{k})\right],
$$

where $I=U / 2$ and $\epsilon(\mathbf{k})$ is the band structure of the system in the absence of the correlation effect, that is, when $U=0$. We have chosen the energy scale so that $\Sigma_{\mathbf{k}} \epsilon(\mathbf{k})=0$. Crystal momentum and also band structure are defined for regions that are covered with Cs and show a local 2D periodic structure. This assumption is based on the STM data. Again for simplicity, we can treat the system as a hexagonal or rectangular lattice for $\theta=0.5$ or $\theta=1$, respectively. Furthermore, for the latter structure the first- and the second-nearest-neighbor transfer integrals are assumed to be equal as in a square lattice. If we know $\rho_{b}(E)$ corresponding to a band structure $\epsilon(\mathbf{k})$, we can find $\rho(E)$ by using the composite function $\rho(E)=\rho_{b}[f(E)]$, where $f(E)=E-I-\left(I^{2} / E-I\right)$. For example, for the $2 \mathrm{D}$ square lattice (which represents the $\theta=1$ structure), $\rho_{b}$ is given by a complete elliptic integral of the first kind. If $U=0, f(E)=E$ and hence $\rho(E)=\rho_{b}(E)$. On the other hand, for $U>0$ independent particle approximation fails, because energy of an electron at a certain site depends upon whether or not this site is already occupied. This repulsive interaction causes the DOS to be separated into two parts, a filled one $\rho_{c, f}$ and an empty one $\rho_{c, e}$; the peak-to-peak energy separation between them is $\delta=U$. The band gap is $\Delta=\sqrt{16 t^{2}+U^{2}}-4 t$, which is smaller than $\delta$. Note that the gap equation is the same for all structures as long as their bandwidths are equal. For the hexagonal structure 
corresponding to $\theta=0.5$, the peak-to-peak energy spacing between $\rho_{c, f}$ and $\rho_{c, e}$ becomes $\delta=\sqrt{16 t^{2}+U^{2}}$. Since there is no well-defined and homogeneous crystal structure of adsorbate between $\theta=0.5$ and $\theta=1$, it is not clear what the DOS looks like. It has been proposed that as Cs coverage increases from half-monolayer coverage, the 0.5 phase (having hexagonal structure) gradually changes into the $\theta=1$ phase [having rectangular structure with the lattice parameters of the ideal $\mathrm{GaAs}(110)$ surface]. ${ }^{16}$ This means that for $\theta<1$ the regions of the $\theta=0.5$ and $\theta=1$ phases coexist, but the extent of the $\theta=1$ phase increases as $\theta \rightarrow 1$. In view of this model it is expected that the DOS curve will follow a rather smooth transition from hexagonal to square lattice type. Apparently, the work by Whitman et al. ${ }^{13}$ points to a different evolution of the adsorbate structure as $\theta \rightarrow 1$.

Since there is always one electron per site for both phases $(\theta=0.5$ and $\theta=1)$, the lower part of the DOS $\left(\rho_{c, f}\right)$ is always filled and therefore the system is insulating as long as $\rho_{c, f}$ and $\rho_{c, e}$ are detached. As $4 t / U$ increases $\rho_{c, f}$ and $\rho_{c, e}$ approach each other, eventually they touch when $4 t / U=1, E_{F}$ passing through the point of contact. However, our approximate solution (the socalled Hubbard-I approximation) which is valid for $t<U$, breaks down during this transition. An improved method (Hubbard-III) (Ref. 20) predicts that the gap between the two pieces of the DOS vanishes when $t / U$ becomes sufficiently large. Further increase of $4 t / U$ ratio causes $\rho_{c, f}$ and $\rho_{c, e}$ to merge into a single DOS with an increased density at $E_{F}$. Therefore, the effect of Coulomb repulsion $U$ is to suppress the DOS at $E_{F}$ and to open a gap between the filled and empty states, if it is large compared to $t$. However, if $U$ is small in comparison to the bandwidth, the DOS is only slightly modified.

The evolution of the DOS in the band gap of the GaAs(110) surface can be related to the coverage of Cs. Regarding the adsorbate structure for $\theta<0.5$, we consider two possibilities. According to the first one, zigzag Cs atoms form islands, which yields a local hexagonal structure. The bandwidth of this structure (at $U=0$ ) can be obtained from the self-consistent pseudopotential calculations using LDA. In the past, the electronic structure of $\mathrm{Na}$ adsorbed on the GaAs(110) surface was studied as a prototype system. The conclusions drawn thereof were extrapolated to other alkali atoms adsorbed on the same surface. Since most of the available pseudopotentials for Cs atoms are not suitable for an accurate description of the electronic structure, the value of $t$ for Cs adsorbed on $\mathrm{GaAs}(110)$ is estimated by calculating the ratio

$$
\eta=\frac{\left\langle\Psi_{\mathrm{Cs}}\left|H_{L D}\right| \Psi_{\mathrm{Cs}}^{\prime}\right\rangle}{\left\langle\Psi_{\mathrm{Na}}\left|H_{L D}\right| \Psi_{\mathrm{Na}}^{\prime}\right\rangle},
$$

where $H_{L D}$ denotes the Hamiltonian in Eq. (1) without the $U$ term. The orbitals $\left|\Psi_{\mathrm{Cs}}\right\rangle$ and $\left|\Psi_{\mathrm{Na}}\right\rangle$ stand for the localized states in Eq. (1) formed between substrate and adsorbate atoms ( $\mathrm{Cs}$ and $\mathrm{Na}$, respectively). The prime corresponds to the nearest-neighbor orbital. Here, we have two limiting cases. In the first one there is no charge transfer from the alkali atom to the surface and therefore the localized states are simply the $6 s$ and $4 s$ or- bitals of $\mathrm{Cs}$ and $\mathrm{Na}$, respectively. By using the value of $t$ for the Na-GaAs(110) system $^{11}$ (which is $\simeq 0.04 \mathrm{eV}$ ) and the atomic orbitals obtained from the Herman-Skillman tables, we calculate $\eta \simeq 2$. 6 within the Hückel approximation and hence $t \simeq 0.1 \mathrm{eV}$ for the Cs-GaAs(110) system. Using the estimate of $U=1.5 \pm 0.3 \mathrm{eV}$ (Ref. 11) we obtain $t / U \simeq 0.06$. In the second limit, there is complete charge transfer from the adsorbate to the surface states. Consequently, the localized states correspond to dangling bonds of $\mathrm{Ga}$ character. Thus, the hopping integral is almost independent of the adsorbate and hence $\eta \simeq 1$ for which $t / U \simeq 0.03$. Accordingly, in both cases, the Hubbard-I approximation, which is valid as long as $t / U$ is sufficiently small, predicts an insulating phase. Furthermore, based on the above discussion one finds $\Delta \simeq 1.5$ $\mathrm{eV}$ and $\delta \simeq 1.5 \mathrm{eV}$. Assuming that $\rho_{c, f}$ is $0.4 \mathrm{eV}$ above the maximum of the valence band, the present model projects a nonmetallic state for $\theta \leq 0.5$, but fails to explain the band-gap narrowing with increasing Cs coverage for $\theta<0.5$. According to the second possibility the structure of the adsorbate is coverage dependent. ${ }^{13}$ At very low coverage, individual chains of Cs have an insulating character with a local band gap of $1.1 \mathrm{eV} .{ }^{13}$ The filled band can split owing to the electron correlation. The empty part of each filled band is expected to overlap with the conduction-band continua. Upon increasing coverage, a third row of Cs atoms is added to the zigzag structure. This increases the width of the filled state, and hence reduces the band gap.

The 2D structure, which occurs at $\theta \sim 0.6$, is different from the $\theta=0.5$ phase having hexagonal structure. The EELS peak occurring at $E_{1}=0.42$ and $E_{2}=1.04 \mathrm{eV}$ for $\theta>0.5$ are in compliance with the compressed phase ${ }^{16}$ or $\theta=1$ phase in registry with the rectangular surface unit cell of GaAs(110) surface. For the compressed phase, $U$ is smaller than that in the $\theta=0.5$ phase since the nearest-neighbor distance of the adsorbates in the former is reduced. In view of the above theory, the EELS peaks $E_{1}$ and $E_{2}$ are associated with the transitions $\rho_{c, f} \rightarrow \rho_{c, e}$ and $\rho_{c, f} \rightarrow$ GaAs conduction-band, respectively. This implies that $\rho_{c, f}$ lies $\sim 0.4 \mathrm{eV}$ above the maximum of the valence band, and $\delta \simeq U \simeq 0.4 \mathrm{eV}$ for the rectangular structure. Figure 1 presents a schematic representation

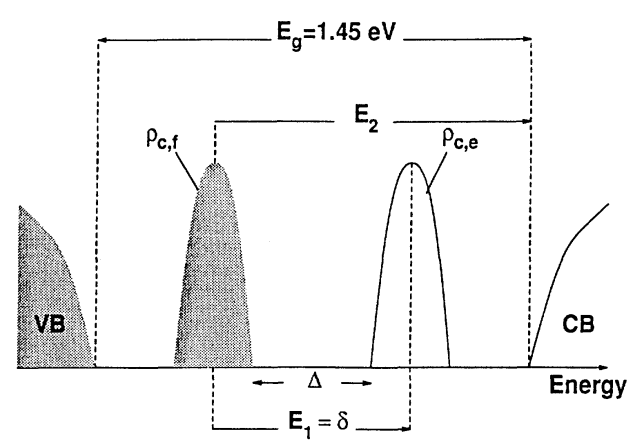

FIG. 1. Schematic description of the density of states in the band gap and the transitions therefrom. $\rho_{c, e}$ and $\rho_{c, f}$ denote the density of states, and $E_{1}$ and $E_{2}$ are the transition energies. 
of these transitions.

Very recently, STM data on K-covered GaAs(110) and InSb(110) and Cs-InSb(110) systems exhibited nonmetallic behavior similar to that of the Cs-covered GaAs(110) discussed above. ${ }^{22}$ By using tunneling $I-V$ curves, Jeon et $a l .{ }^{23}$ also observed the absence of metallicity for the Na-induced $\mathrm{Si}(111)-(3 \times 1)$ surface with $\frac{2}{3} \mathrm{Na}$ coverage, which has unpaired electrons in the unit cell. These findings bring about a new aspect of alkali-metal-covered semiconductor surfaces having unpaired electrons. That is the Mott insulating behavior and support the model given above.
In conclusion, using the Hubbard model we investigated the electronic structure of the Cs-covered GaAs(110) surface at submonolayer coverage. The electron hopping and electron repulsion energy estimated from selfconsistent field calculation indicate that the correlation effects are important and cause the metallic density of states in the band gap to split. As a result the system becomes a Mott-Hubbard insulator with the absent density of states at the Fermi level up to monolayer coverage of Cs. Using an approximate solution of the model, we provided an interpretation of electron-energy-loss and scanning tunneling spectroscopies.
${ }^{1}$ H. Tochiara, Surf. Sci. 126, 523 (1983); T. Aruga, H. Tochiara, and Y. Murata, Phys. Rev. Lett. 53, 372 (1984).

${ }^{2}$ S. Ciraci and I. P. Batra, Phys. Rev. Lett. 56, 877 (1986); ibid. 58, 1982 (1987); ibid. 60, 547 (1988); Phys. Rev. B 37, 2995 (1988).

${ }^{3}$ I. P. Batra, Phys. Rev. B 43, 12322 (1991).

${ }^{4}$ T. Kendelewicz, P. Soukiassian, R. S. List, J. C. Woicik, P. Pianetta, I. Lindau, and W. E. Spicer, Phys. Rev. B 37, 7115 (1988).

${ }^{5}$ E. M. Oellig, E. G. Michel, M. C. Asensio, R. Miranda, J. C. Duran, A. Munoz, and F. Flores, Europhys. Lett. 5, 727 (1988).

${ }^{6}$ Metallization and Metal-Semiconductor Interfaces, edited by I. P. Batra (Plenum, New York, 1989).

${ }^{7}$ I. P. Batra, E. Tekman, and S. Ciraci, Prog. Surf. Sci. 36, 289 (1991).

${ }^{8}$ I. P. Batra and S. Ciraci, Phys. Rev. B 37, 8432 (1988).

${ }^{9}$ C. Y. Fong, L. H. Yang, and I. P. Batra, Phys. Rev. B 40, 6120 (1989).

${ }^{10}$ J. Ortega and F. Flores, Phys. Rev. Lett. 63, 2500 (1989).

${ }^{11}$ J. Hebenstreit, M. Heinemann, and M. Scheffler, Phys. Rev. Lett. 67, 1031 (1991).

${ }^{12}$ P. N. First, R. A. Dragoset, J. A. Stroscio, R. J. Celotta, and
R. Feenstra, J. Vac. Sci. Technol. 7, 2868 (1989).

${ }^{13}$ L. J. Whitman, J. A. Stroscio, R. A. Dragoset, and R. J. Celotta, Phys. Rev. Lett. 66, 1338 (1991).

${ }^{14}$ K. O. Magnusson and B. Reihl, Phys. Rev. B 40, 7814 (1989).

${ }^{15}$ T. M. Wong, N. J. DiNardo, D. Heskett, and E. W. Plummer, Phys. Rev. B 41, 12342 (1990).

${ }^{16}$ N. J. DiNardo, T. M. Wong, and E. W. Plummer, Phys. Rev. Lett. 65, 2117 (1990).

${ }^{17}$ N. F. Mott, Rev. Mod. Phys. 40, 667 (1968).

${ }^{18}$ A. Ferraz, N. H. March, and F. Flores, J. Phys. Chem. Solids 45, 627 (1984).

${ }^{19}$ J. Hubbard, Proc. R. Soc. London A276, 238 (1963).

20J. Hubbard, Proc. R. Soc. London A281, 401 (1964); for further discussion, see P. Fulde, Electron Correlations in Molecules and Solids (Springer-Verlag, Berlin, 1991).

${ }^{21}$ J. E. Hirsch, Phys. Rev. Lett. 54, 1317 (1985).

${ }^{22}$ L. J. Whitman, Joseph A. Stroscio, R. A. Dragoset, and R. J. Celotta, in Proceedings of the NATO Advanced Study Institute Conference on Atomic and Nanoscale Modifications of Materials, edited by $\mathrm{Ph}$. Avouris (Kluwer, Dordrecht, 1993).

${ }^{23}$ D. Jeon, T. Hashizume, T. Sakurai, and R. F. Willis, Phys. Rev. Lett. 69, 1419 (1992). 